ISSN 0258-7122 (Print), 2408-8293 (Online)

Bangladesh J. Agril. Res. 44(2): 223-238, June 2019

\title{
DYNAMICS OF STARCH SYNTHESIS ENZYMES AND THEIR RELATIONSHIP WITH CHALKINESS OF EARLY INDICA RICE UNDER DIFFERENT POST-ANTHESIS TEMPERATURE REGIMES
}

\author{
MOHAMMED HUMAYUN KABIR ${ }^{1,2}$, QING LIU $^{1}$, SHITOU XIA ${ }^{1}$ \\ RUOZHONG WANG ${ }^{1}$ AND LANGTAO XIAO ${ }^{1}$
}

\begin{abstract}
An experiment on an early indica rice cv. 'Shenyou9576' was conducted in the Key Laboratory of Phytohormones and Growth Development of Hunan Agricultural University, Changsha, Hunan, PR China in 2014 to investigate the influence of varying post-anthesis temperatures on chalkiness rate, head rice rate, and on major 6 starch synthesis enzymes i.e., SuSy (EC 1.9.3.1), ADPGPpas (EC 2.7.7.27), SSS (EC 2.4.1.21) and GBSS, (EC 2.4.1.21), SBE (EC 2.4.1.18) and SDBE (EC 3.2.1.70). The treatments comprised of three temperature regimes which are designated as the high $\left(35 / 28^{\circ} \mathrm{C}\right.$ - day/night), low $\left(25 / 20^{\circ} \mathrm{C}\right.$ - day/night) and natural condition $\left(35 / 25^{\circ} \mathrm{C}\right.$-day/night $)$ as the control. Under high temperature maximum chalkiness rate was $61.11 \%$ and minimum was $22.59 \%$ under low temperature treatment. The lowest head rice rate was $42.76 \%$ under high temperature treatment followed by $49.91 \%$ in the control, while the highest rate was $62.33 \%$ under low temperature treatment. Maximum grain filling rate (Gmax) was found highest (1.69 $\mathrm{mg} /$ day) in the high temperature and average grain filling rate (Gavg) was found highest (1.36 $\mathrm{mg} /$ day) under the control. The activity of SuSy, ADPG-Ppase, SSS and GBSS were decreased gradually from 14 to 35 days after flowering (DAF). Irrespective of the treatments, an increasing trend of ADPG-Ppase activity was observed from 7 to $14 \mathrm{DAF}$ and then declined. Correlation between the chalkiness and the enzymes activity of SuSy, ADPG-Ppase and SSS were significantly negative at 21, 28 and 35 DAFs, i.e., higher activity of SuSy, ADPG-Ppase and SSS at the mid-late to the late caryopsis development stage mediated by low temperature treatment played an important role for the reduction of chalkiness. The correlation between GBSS activity and chalkiness was significantly negative and stronger at 14, 21 and 28 DAF indicating that GBSS played a cardinal role to reduce chalkiness in the mid to mid-late stage of rice grain development. Significantly negative correlation was found between starch branching enzyme (SBE) and chalkiness at 21, 28 and 35 DAF, i.e., the higher SBE activity under low temperature treatment at the later grain filling stage also had a positive role in reduction of chalkiness.
\end{abstract}

Keywords: Rice (Oryza sativa L.), high temperature, starch synthesis enzymes, head rice rate, and chalkiness rate.

${ }^{1}$ Hunan Provincial Key Laboratory of Phytohormones and Growth Development, Hunan Agricultural University and Southern Regional Collaborative Innovation Center for Grain and Oil Crops in China, Changsha 410128, Hunan, China, ${ }^{2}$ Adaptive Research Division, Bangladesh Rice Research Institute, Gazipur-1701, Bangladesh. 


\section{Introduction}

Rice (Oryza sativa L.) is one of the major cereal crops that are produced worldwide and the staple food for more than half of the world's population. Grain quality, next to yield, is the most important factor in rice production and in economic returns for farmers. The most important rice quality components, common to all consumers, include appearance, milling, cooking, eating and nutrient qualities (Koutroubas et al., 2004). Appearance of rice grain is an important quality character which depends on endosperm color and dimension. Endosperm color is mainly determined by the degree of chalkiness, i.e., the white opaque part in rice endosperm. Rice grains with more chalkiness are likely to break during milling, which affects the commercial value of milled rice and the market acceptability (Borrell et al., 1997). Besides, rice grain with more chalkiness influences on cooking, nutritional and eating quality. The hazard of high temperature is serious to the forming of grain quality in rice during grain filling period. Previous studies showed that the environmental (specially daily average temperature during grain filling) and genetic factors are associated with chalkiness formation (Borrell et al., 1997; Dong et al., 2006; Koutroubas et al., 2004; Zhang et al., 2008).

Accumulation of starch in rice grains is the end process of photosynthesis. The sink strength can be described as the product of sink size and sink activity (Venkateswarlu et. al., 1987). Sucrose in the grains eventually becomes starch through a series of enzymatic reactions (Douglas et. al., 1988; Keeling et. al., 1988). It has been well documented that rice quality is mainly determined by starch composition (Han and Hamaker., 2001). Therefore, biochemical processes of starch synthesis, i.e., the activity level of starch synthesis related enzymes in the grains determine rice grain quality as well as play an important role on chalkiness formation and grain weight. It has been shown that major six enzymes are involved in the conversion of sucrose into starch in rice endosperms, including sucrose synthase (SuSy; EC 1.9.3.1), ADP-glucose pyrophosphorylase (ADPG-Ppase; EC2.7.7.27), soluble starch synthase (SSS, EC 2.4.1.21) and granule bound starch synthase (GBSS, EC 2.4.1.21), starch branching enzyme (SBE; EC2.4.1.18) and starch de-branching enzyme (SDBE; EC3.2.1.70) (Nakamura et.al., 1989; Kubo et.al., 1999). Although many studies have been carried out on the effect of high temperature on chalkiness, starch synthesis and others in rice, but little is known about the low temperature and integrated information of different temperature regimes on chalkiness, head rice rate, starch synthesis enzymes activities, at grain filling stage of early indica rice. The experiment was, therefore conducted to investigate the influence of varying postanthesis temperature on chalkiness, head rice rate, and major starch synthesis related enzymes in rice grains. 


\section{Materials and Methods}

\section{Experiment description}

The experiment was carried out in the Key Laboratory of Phytohormones and Growth Development of Hunan Agricultural University, Changsha, Hunan, PR China, in 2014. The tested rice variety was heat tolerant an early indica rice 'Shenyou 9576'. Rice seeds were washed thoroughly by distilled water and then placed on wetted blotter paper in petridishes for germination. Germinated seeds of rice were pre-grown with complete Kimura B nutrient solution (Yoshida et al., 1976) in a greenhouse for 15 days. Seedlings were then transferred to earthen pots of $30 \mathrm{~cm}$ in diameter and $32 \mathrm{~cm}$ in depth filled with $7.0 \mathrm{~kg}$ of sieved, dry paddy soil (the contents of soil: organic matter, alkaline hydrolytic nitrogen, effective phosphorus, available potassium were $1.8 \%, 66.2 \mathrm{mg} \mathrm{kg}^{-1}, 8.5 \mathrm{mg} \mathrm{kg}^{-1}$, and $8.0 \mathrm{mg} \mathrm{kg}^{-1}$, respectively, and soil $\mathrm{pH}$ was 5.4) amended with $1.0 \mathrm{~g}$ $\mathrm{CO}\left(\mathrm{NH}_{2}\right)_{2}, 0.4 \mathrm{~g} \mathrm{P}_{2} \mathrm{O}_{5}$, and $0.6 \mathrm{~g} \mathrm{~K}_{2} \mathrm{O}$ per $\mathrm{kg}$ soil to grow. Three earthen pots were placed in different growth chamber as well as in the net house. And two seedlings were transplanted in each earthen pot. Proper management practices were provided as per requirement for proper growth of the seedlings. The treatment consisted of three temperature regimes which are designated as high temperature $\left(35 / 28^{\circ} \mathrm{C}, 12 \mathrm{~h}\right.$ light $/ 12 \mathrm{~h}$ dark, $75-80 \%$ relative humidity), low temperature $\left(25 / 20^{\circ} \mathrm{C}, 12 \mathrm{~h}\right.$ light $/ 12 \mathrm{~h}$ dark, $75-80 \%$ relative humidity) and natural condition $\left(35 / 25^{\circ} \mathrm{C}\right.$-day/night) as control. The treatments were imposed after anthesis by transferring pots into different growth chambers, but for the control treatment pots were kept in the net house under natural condition. The experiment was laid out in a complete randomized design (CRD) with three replications.

\section{Sampling method}

Panicles with rice grains of each treatment were collected at 7 day interval after flowering i.e. 7, 14, 21, 28 and 35 days after flowering (DAF). Samples were collected between 9.00 to 11.00 am and immediately wrapped in aluminum foil and frozen in liquid nitrogen, then placed into a sealed plastic bag and stored at $60^{\circ} \mathrm{C}$ until used for different analysis. Rice grains were harvested at $35 \mathrm{DAF}$ and then were sun dried to achieve 14\% moisture content. Rough rice (paddy rice) was dehusked by a SBS- 80 dehuller, then was polished by a rice polisher for 2 minutes. Milled rice samples were kept in sealed bags under refrigeration $\left(4^{\circ} \mathrm{C}\right)$ for later analysis.

\section{Chalkiness and head rice rate measurement}

Chalkiness was measured with a system composed of a scanner and a special software Chalkiness 2.0 developed by Hunan Agricultural University (Chen et al., 2011). Head rice refers to the whole grains of milled rice and was computed by using the following equation (Gummert, 2010). 


$$
\text { Head rice }(\%)=\frac{\text { Wt of whole grains }}{\text { Wt of paddy samples }} \times 100
$$

\section{Grain filling rate}

The grain filling process was fitted by the growth equation of Richards (1959) as described by Zhu et.al. (1988).

$$
W=A /\left(1+B e^{-k t}\right)^{-\frac{2}{N}}
$$

Grain filling rate $(\mathrm{G})$ was calculated as the derivative of equation 1

$$
\mathrm{G}=\mathrm{AKBe}^{-\mathrm{kt}} /\left(\mathrm{N}\left(1+\mathrm{Be}^{-\mathrm{kt}}\right)^{(\mathrm{N}+1) / \mathrm{N})}\right.
$$

where $\mathrm{W}$ is the grain weight $(\mathrm{mg}), \mathrm{A}$ is the final grain weight $(\mathrm{mg}), \mathrm{t}$ is the time after anthesis (day), and $\mathrm{B}, \mathrm{k}$, and $\mathrm{N}$ are coefficients determined by regression. The active grain filling period was defined as that when $\mathrm{W}$ was from $5 \%(\mathrm{t} 1)$ to 95\% ( $\mathrm{t} 2$ ) of $\mathrm{A}$. The average grain filling rate during this period was calculated from t 1 to $\mathrm{t} 2$. Average grain filling rate $\left(\mathrm{G}_{\mathrm{avg}} ; \mathrm{mg} / \mathrm{day}\right)$, maximum grain filling rate $\left(\mathrm{G}_{\max } ; \mathrm{mg} /\right.$ day $)$, time of maximum grain filling $\left(\mathrm{T}_{\max . \mathrm{G}}\right.$; day), weight at maximum grain filling time $\left(\mathrm{W}_{\text {max.G }} ; \mathrm{mg}\right)$, growth $\%$ of the final growth at the time of maximum grain filling time $(\mathrm{I} \%)$, initial growth potential $(\mathrm{Ro}=\mathrm{K} / \mathrm{N})$, grain filling time (day) were calculated from the equation 2.

\section{Starch synthesis enzymes assay procedures}

\section{Preparation of enzymes extraction}

The preparation procedure was similar to the method of Nakamura et al., (1989). Three rice grains were de-hulled and then separated from embryo and pericarp. Weight of 3 grains was recorded and then hand-homogenized with a pestle in a pre-cooled mortar (at 0-2 ${ }^{\circ} \mathrm{C}$ ) with $1 \mathrm{~mL}$ of ice-cooled GS buffer (extraction buffer) containing $100 \mathrm{mM}$ Hepes-NaOH, (pH 7.4), $8 \mathrm{mM} \mathrm{MgCl} 2,2 \mathrm{mM}$ $\mathrm{K}_{2} \mathrm{HPO}_{4}, 2 \mathrm{mM} \mathrm{Na} 2$-EDTA, 12.5\% (V/V) Glycerol, 5\% (W/V) PVP-40, $50 \mathrm{mM}$ 2-mercaptoethanol. After grinding, the liquid was transferred into a $2 \mathrm{~mL}$ tube, and then centrifuged at $2^{\circ} \mathrm{C}, 5000 \mathrm{rev} / \mathrm{min}$ for 10 minutes. The supernatant portion was transferred into another tube and GS-buffer was added to the sediment, and the operation was repeated again. The supernatant and the sediment were collected separately. The resulting supernatant was used for sucrose synthase (SuSy; EC 1.9.3.1), ADP-glucose pyrophosphorylase (ADPGPpase; EC2.7.7.27), soluble starch synthase (SSS, EC 2.4.1.21), starch branching enzyme (SBE; EC2.4.1.18) and starch de-branching enzyme (SDBE; EC3.2.1.70) and the pellet portion was used for granule bound starch synthase (GBSS, EC 2.4.1.21) enzyme assay. Three replications were done for every experiment. 


\section{Enzymes assay}

The assay of ADP-glucose pyrophosphorylase (ADPG-Ppase; EC2.7.7.27), soluble starch synthase (SSS, EC 2.4.1.21) and starch branching enzyme (SBE; EC2.4.1.18) or Q-enzyme were similar to the procedure of Nakamura et al. (1989). Sediment or pellet was used instead of crude enzyme extraction for the assay of granule bound starch synthase (GBSS, EC 2.4.1.21) and the procedure is same to SSS. The assay of sucrose synthase (SuSy; EC 1.9.3.1), was carried out according to the protocol of Wardlaw and Willenbrink (1994). Starch debranching enzyme (SDBE; EC3.2.1.70) or R-enzyme activity was assayed according to the protocol of Nelson (1944) and Somogyi (1952). All the assays were carried out at $30^{\circ} \mathrm{C}$. Assays were conducted in the range of concentrations of enzyme where the activity increased linearly with increases in the amount of enzyme preparation and the reaction time. The background values were routinely taken as the activities detected with a reaction time of zero (the enzymes were denatured immediately after their addition to the reaction mixtures). One unit of activity of the above enzymes was defined as the amount causing an increase in absorbance of one unit at 340,480, 520 and $540 \mathrm{~nm}$ in one min. All the spectrophotometer readings were taken by using a PGENERAL T6 XinRui spectrophotometer (Persee company, Beijing 101200, China). The activities of ADPG-Ppase, SSS, GBSS were defined as NADPH amount by measuring the increase in absorbance at $340 \mathrm{~nm}$ and SBE activity was defined as amount of $1 \%$ KI-I $\mathrm{I}_{2}$ decreasing at $540 \mathrm{~nm}$. The chemical products were purchased from SigmaAldrich, Ruibio and others chemical companies.

\section{Statistical analysis}

All experimental data were analyzed following analysis of variance. All statistical analysis was performed by using a statistical software, DPS version 12.01. Mean separation of the treatments was done by using Least Significant Difference (LSD) test at 5\% level. Microsoft Excel 2003 (Microsoft, USA) was used to generate graphs.

From flowering to harvest, under the natural condition (control), the daily average temperature of the experimental site was within $25^{\circ} \mathrm{C}$ to $35^{\circ} \mathrm{C}$.

\section{Results and Discussion}

\section{A. Results}

Changes of chalkiness rate and head rice rate under different temperature regimes:

Chalkiness (\%) was significantly affected by different temperature regimes (Fig. 1a). The highest chalkiness rate $(61.11 \%)$ was observed under high temperature and the lowest $(22.59 \%)$ under low temperature, whereas in control, chalkiness rate was in between high and low temperature, which was $47.81 \%$. Therefore, 
chalkiness was increased by high temperature stress at the grain filling stage. The highest head rice was $62.33 \%$ under low temperature followed by $49.91 \%$ under the control and the lowest (42.76\%) under high temperature (Fig. 1b). Therefore, the results indicate that high temperature stress had a negative effect on head rice $(\%)$, while low temperature had a positive effect.
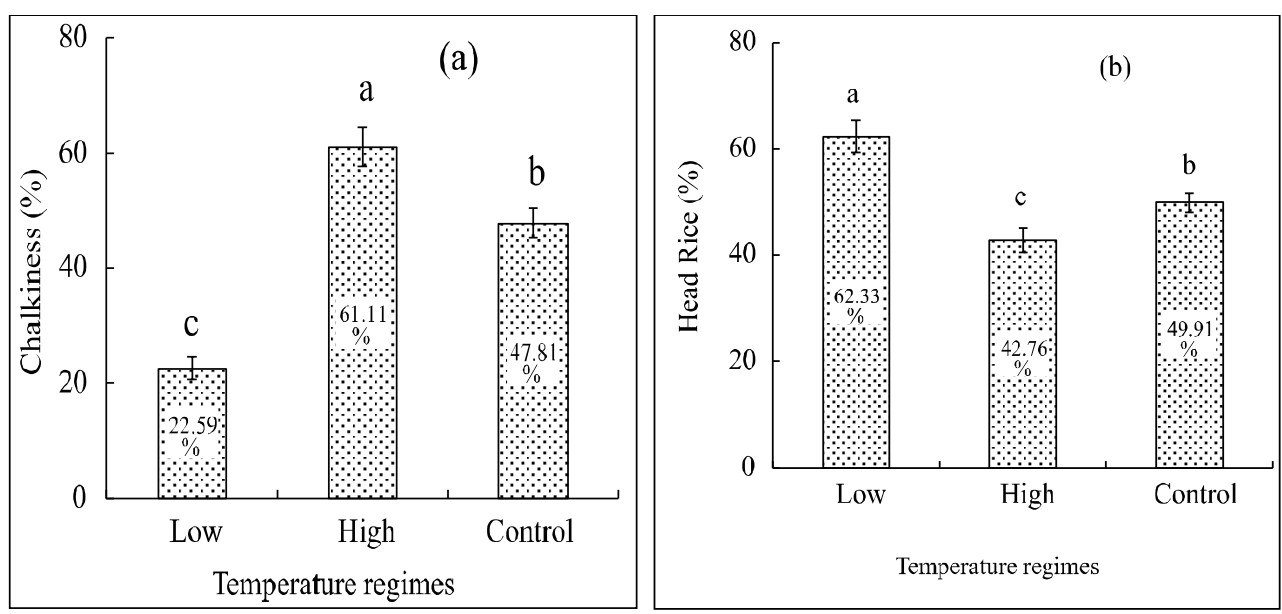

Fig. 1. (a) Chalkiness (\%) and (b) head rice (\%) of an early indica rice cv. 'Shenyou 9576' as influenced by temperature regimes after flowering. Vertical bars represent $\pm \mathrm{SE}$ of the mean $(n=3)$.

\section{Enzymes activities in rice endosperm under different temperature regimes}

\section{Activity of SuSy (EC 2.4.1.13) in rice endosperm}

The differences among the treatments on SuSy activity in rice endosperm were remarkable at significant level throughout the grain filling period (Fig. 2). The SuSy activity showed single-peak curve in all the three temperature treatments during grain filling period. At 7 DAF the highest peak (48.46 nmol.endosperm $\left.{ }^{1} . \mathrm{min}^{-1}\right)$ was observed under high temperature treatment followed by the control (43.8 nmol.endosperm ${ }^{-1} \cdot \mathrm{min}^{-1}$ ) and after that SuSy activity decreased sharply up to 28 DAF compared with low temperature treatment. On the other hand, the highest peak (42.69 nmol.endosperm ${ }^{-1} \cdot \mathrm{min}^{-1}$ ) was observed at $14 \mathrm{DAF}$ in the low temperature treatment. From 14 DAF the highest activity was observed under low temperature treatment followed by the control and high temperature treatment.

\section{Activity of ADPG-Ppase (EC 2.7.7.27) in rice endosperm}

The dynamic changes of the activity of ADPG-Ppase under three temperature treatments at the grain filling stage is shown in Fig. 3. At 7 DAF, the highest activity of ADPG-Ppase (18.78 nmol.endosperm $\left.{ }^{-1} \cdot \mathrm{min}^{-1}\right)$ was observed under high temperature treatment followed by the control $\left(15.86\right.$ nmol.endosperm $\left.{ }^{-1} \cdot \mathrm{min}^{-1}\right)$, 
whereas the lowest (11.94 nmol.endosperm $\left.{ }^{-1} \cdot \mathrm{min}^{-1}\right)$ was observed in the low temperature treatment. An increasing trend of the enzyme was observed for all the three temperature treatments from 7 to $14 \mathrm{DAF}$. Irrespective of treatments the maximum ADPG-Ppase activity was displayed on 14 DAF after that, ADPG-Ppase activity was declined. At $14 \mathrm{DAF}$, the highest $\left(22.34 \mathrm{nmol}\right.$.endosperm $\left.{ }^{-1} \cdot \mathrm{min}^{-1}\right)$ activity was observed under low temperature treatment followed by high temperature treatment $\left(20.43 \mathrm{nmol}\right.$.endosperm $\left.{ }^{-1} \cdot \mathrm{min}^{-1}\right)$ and the lowest activity (19.34 nmol.endosperm ${ }^{-1} \cdot \mathrm{min}^{-1}$ ) was observed in the control. From 14 DAF the highest activity was found under low temperature treatment and from 21 DAF the second highest activity was found under the control and the lowest activity was observed under high temperature treatment.

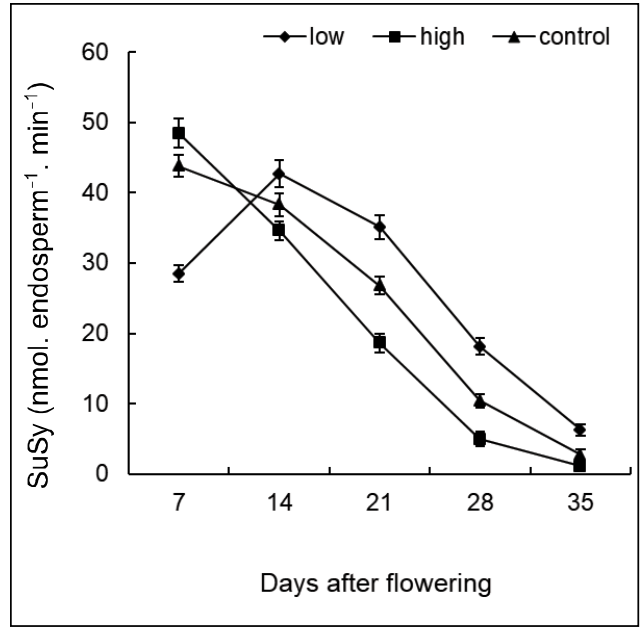

Fig. 2. Changes of SuSy activity in developing rice grain. Vertical bars represent $\pm \mathrm{SE}$ of the mean $(n=3)$.

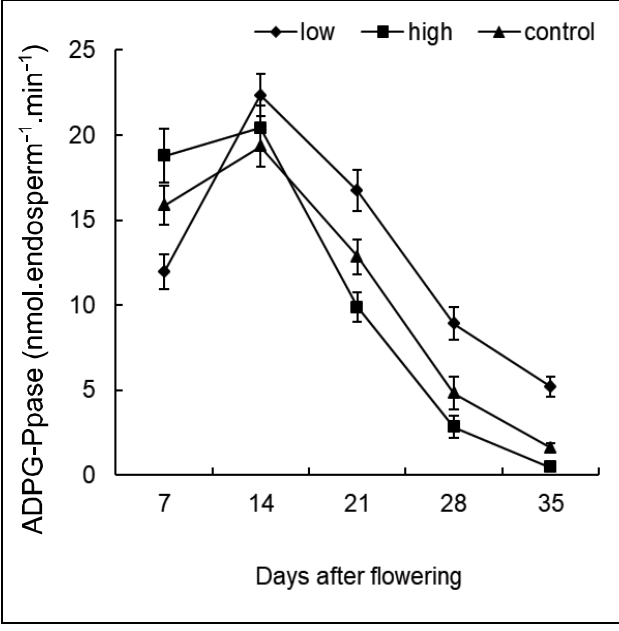

Fig. 3. Changes of ADPG-Ppase activity in developing rice grain. Vertical bars represent $\pm \mathrm{SE}$ of the mean $(\mathbf{n}=\mathbf{3})$.

\section{Activity of SSS (EC 2.4.1.21) in rice endosperm}

Temperature treatments showed significant influence on SSS activity in rice endosperm as shown in Fig. 4. Over the grain filling period, the SSS activity in the three temperature treatments exhibited single peak curves. Maximum values of SSS was found at 7 DAF under high temperature treatment (7.74 nmol.endosperm $\left.{ }^{-1} \cdot \mathrm{min}^{-1}\right)$ and the control $\left(6.4 \mathrm{nmol}\right.$.endosperm $\left.{ }^{-1} \cdot \mathrm{min}^{-1}\right)$. But, under low temperature treatment, the highest SSS activity was 5.07 nmol.endosperm ${ }^{-1} \cdot \mathrm{min}^{-1}$ at $14 \mathrm{DAF}$. Moreover, among the three temperature treatments the highest SSS activity was found under low temperature treatment from 21 to 35 DAF, i.e., under low temperature treatment SSS activity was lower at the beginning but higher at the middle and late grain filling stages. However, SSS activity under high temperature treatment showed a quick decreasing trend 
from 7 to 28 DAF. But, SSS activity in the control showed a slow decreasing trend from 7 to 28 DAF.

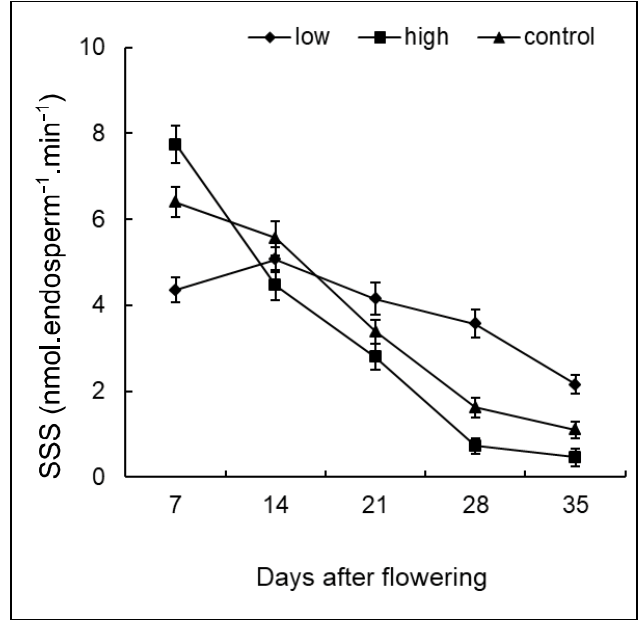

Fig. 4. Changes of SSS activity in developing rice grain. Vertical bars represent $\pm \mathrm{SE}$ of the mean $(n=3)$.

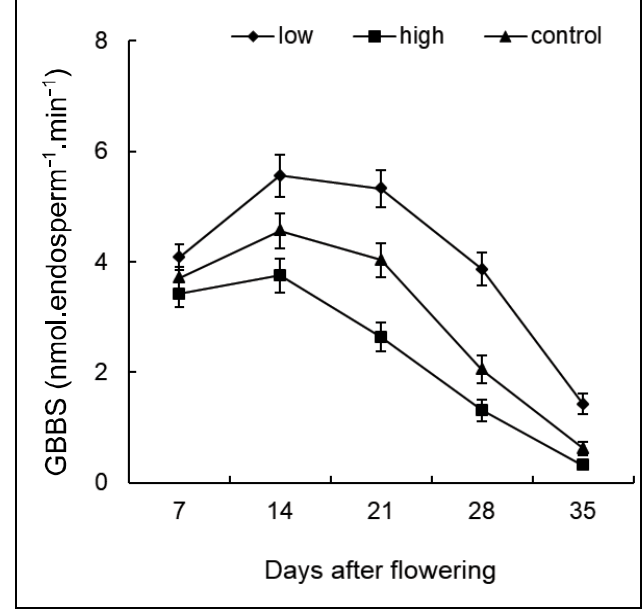

Fig. 5. Changes of GBSS activity in developing rice grain. Vertical bars represent $\pm \mathrm{SE}$ of the mean $(\mathbf{n}=3)$.

\section{Activity of GBSS (EC 2.4.21) in rice endosperm}

The dynamic changes of GBSS enzyme throughout the grain filling period are shown in Fig. 5. Irrespective of the temperature treatments an increasing trend of GBSS activity was observed from 7 DAF to 14 DAF and then declined. Among the temperature treatments the highest GBSS activity was found in the low temperature treatment throughout the grain filling period. The highest GBSS activity was $5.56 \mathrm{nmol}$.endosperm ${ }^{-1} \cdot \mathrm{min}^{-1}$ at $14 \mathrm{DAF}$ under low temperature treatment, reduced to $1.43 \mathrm{nmol}$.endosperm ${ }^{-1} \cdot \mathrm{min}^{-1}$ at $35 \mathrm{DAF}$, while, at $14 \mathrm{DAF}$ under high temperature treatment GBSS activity was the lowest which was 3.75 nmol.endosperm ${ }^{-1} \cdot \mathrm{min}^{-1}$, reduced to $0.32 \mathrm{nmol}$.endosperm ${ }^{-1} \cdot \mathrm{min}^{-1}$ at $35 \mathrm{DAF}$. Among the treatments, variation of GBSS activity in rice endosperm was maximum at $21 \mathrm{DAF}$ and was minimum at 7 DAF.

\section{Activity of SBE or Q-enzyme (EC 2.4.1.18) in rice endosperm}

The effect of temperature on SBE activity in rice endosperm was significant throughout the grain filling period (Fig. 6). Relative to the control, the highest SBE activity (15.76 nmol.endosperm $\left.{ }^{-1} \cdot \mathrm{min}^{-1}\right)$ was found under high temperature treatment and the lowest was $\left(8.18 \mathrm{nmol}\right.$. endosperm $\left.{ }^{-1} \cdot \mathrm{min}^{-1}\right)$ found under low temperature treatment at $7 \mathrm{DAF}$. SBE activity both in high temperature treatment and the control increased from 7 to 14 DAF and then decreased slowly up to 21 $\mathrm{DAF}$, after that SBE activity decreased quickly up to $35 \mathrm{DAF}$. Whereas, under 
low temperature treatment SBE activity jumped from 7 to 14 DAF and exhibited the highest $\left(18.98 \mathrm{nmol} \cdot \mathrm{endosperm}^{-1} \cdot \mathrm{min}^{-1}\right)$ activity at $14 \mathrm{DAF}$ then declined slowly to $7.78 \mathrm{nmol}$.endosperm ${ }^{-1} \cdot \mathrm{min}^{-1}$ at $35 \mathrm{DAF}$. The result suggest that exposure to high temperature increased the SBE activity at the early to the mid grain filling period but became lower at the later grain filling period. The contrary result was observed in case of low temperature treatment.

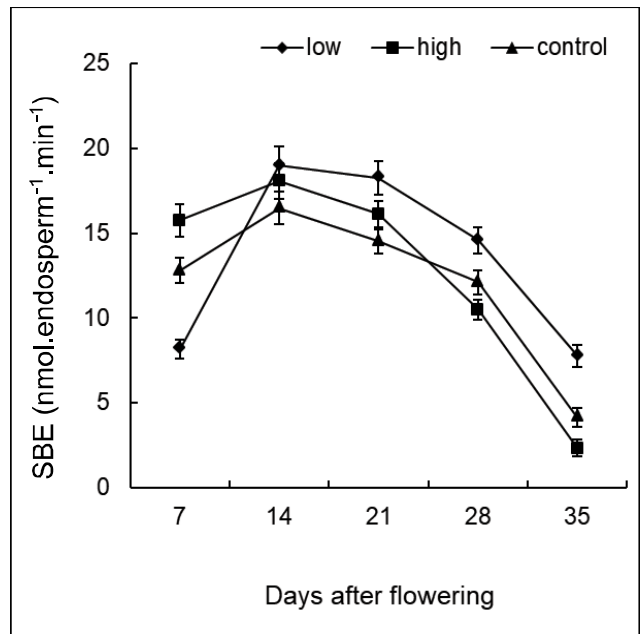

Fig. 6. Changes of SBE or Q-enzyme activity in developing rice grain. Vertical bars represent $\pm S E$ of the mean $(n=3)$.

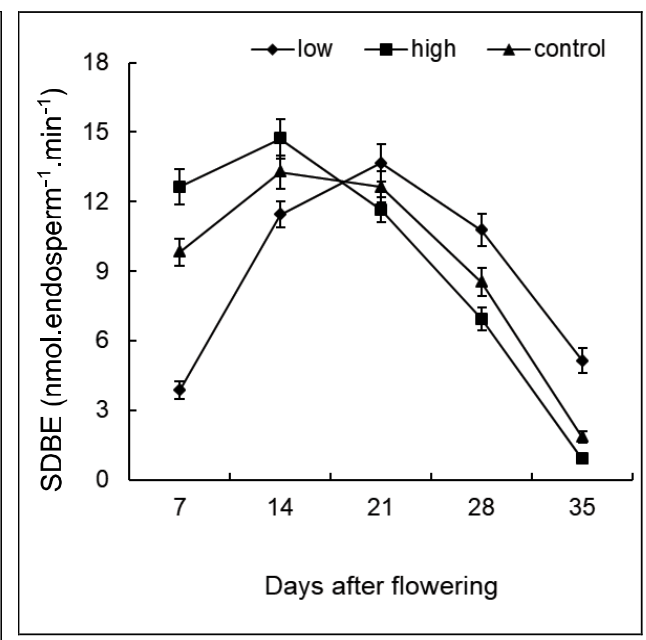

Fig. 7. Changes of SDBE or R-enzyme activity in developing rice grain. Vertical bars represent $\pm \mathrm{SE}$ of the mean $(n=3)$.

\section{Activity of SDBE or R-enzyme (EC 3.2.1.70) in rice endosperm}

The differences among the treatments were distinct at significant level in respect of SDBE activity in all the studied DAFs (Fig. 7). At 7 DAF, high temperature treatment showed significantly the highest SDBE activity $(12.63 \mathrm{nmol}$. endosperm $\left.{ }^{-1} \mathrm{~min}^{-1}\right)$, then increased gradually and had a peak (14.71 nmol. endosperm $\left.{ }^{-1} \cdot \mathrm{min}^{-1}\right)$ at $14 \mathrm{DAF}$. Afterwards, SDBE decreased quickly up to 35 DAF. While, at 7 DAF the second highest SDBE activity $(9.83 \mathrm{nmol}$. endosperm $\left.{ }^{1} \cdot \mathrm{min}^{-1}\right)$ was observed in the control and increased quickly having a peak (13.27 nmol.endosperm $\left.{ }^{-1} \cdot \mathrm{min}^{-1}\right)$ at $14 \mathrm{DAF}$ and then declined. At 7 DAF among the treatments, low temperature treatment showed the lowest SDBE activity (9.83 nmol. endosperm ${ }^{-1} \cdot \mathrm{min}^{-1}$ ) but the highest SDBE activity (13.67 nmol. endosperm ${ }^{1} \cdot \mathrm{min}^{-1}$ ) was found at $21 \mathrm{DAF}$, implying that low temperature was not favourable for SDBE activity at the early to middle stage of grain filling.

\section{Correlation between enzymes activity and chalkiness (\%)}

The correlations between SuSy, ADPG-Ppase, SSS, GBSS, SBE, SDBE, and chalkiness were analyzed and the results were presented in Table 1. The 
correlation between different enzymes activities with chalkiness (\%) varied with the three temperature treatments. The activity of SuSy was positively and significantly associated with chalkiness (\%) only at $7 \mathrm{DAF}$, but significantly and negatively correlated with chalkiness $(\%)$ from 14 to 35 DAF. There was a significant positive correlation between ADPG-Ppase activity and chalkiness (\%) in rice endosperm at $7 \mathrm{DAF}$ but no significant correlation was found at $14 \mathrm{DAF}$, while significantly negative and stronger correlation was observed at 21, 28 and 35 DAF. The activity of SSS was positive and significantly associated with chalkiness $(\%)$ only at $7 \mathrm{DAF}$, but significantly and negatively correlated with chalkiness (\%) from 21 to 35 DAF. GBSS activity exhibited negative correlation with chalkiness $(\%)$ throughout the grain filling period and the relationship was significantly stronger in the 14, 21 and $28 \mathrm{DAF}$, where relationship was weaker at 7 and 35 DAF. Coefficient of correlation between the SBE activity and chalkiness (\%) was significantly positive and stronger at 7 DAF, but significantly negative at 21, 28 and $35 \mathrm{DAF}$ and insignificant correlation was found at 14 DAF. At 7 DAF, the relationship between SDBE and chalkiness (\%) was positively significant and stronger but at the 14 and 21 DAF SDBE activity did not have significant correlation with chalkiness (\%), whereas at 28 and 35 DAF SDBE activity exhibited significant negative correlation with chalkiness (\%).

Table 1. Coefficient of correlation between chalkiness (\%) and enzymes activity

\begin{tabular}{l|c|c|c|c|c}
\hline & $7 \mathrm{DAF}$ & $14 \mathrm{DAF}$ & $21 \mathrm{DAF}$ & $28 \mathrm{DAF}$ & $35 \mathrm{DAF}$ \\
\hline SuSy & $0.8793^{* *}$ & $-0.6733^{*}$ & $-0.8602^{* *}$ & $-0.8386^{* *}$ & $-0.8105^{* *}$ \\
ADPG-Ppase & $0.7261^{*}$ & $-0.5643^{\mathrm{NS}}$ & $-0.9027^{* *}$ & $-0.9237 * *$ & $-0.8703^{* *}$ \\
SSS & $0.8407 * *$ & $-0.4857^{\mathrm{NS}}$ & $-0.7265^{*}$ & $-0.8808^{* *}$ & $-0.8180^{* *}$ \\
GBSS & $-0.7253^{*}$ & $-0.8899^{* *}$ & $-0.9141^{* *}$ & $-0.8737^{*} *$ & $-0.7406^{*}$ \\
SBE & $0.9204^{*} *$ & $-0.4651^{\mathrm{NS}}$ & $-0.6715^{*}$ & $-0.6978^{*}$ & $-0.8107^{* *}$ \\
SDBE & $0.9029^{*}$ & $0.6433^{\mathrm{NS}}$ & $-0.5384^{\mathrm{NS}}$ & $-0.7514^{*}$ & $-0.8496^{* *}$ \\
\hline
\end{tabular}

Note: $* *$, $*$ and NS indicate significant differences at $1 \%, 5 \%$ probability level, and nonsignificant, respectively.

\section{Starch accumulation parameters}

Starch accumulation parameters e.g. average grain filling rate $\left(\mathrm{G}_{\mathrm{avg}} ; \mathrm{mg} / \mathrm{day}\right)$, maximum grain filling rate $\left(\mathrm{G}_{\max } ; \mathrm{mg} /\right.$ day $)$, initial growth potential $(\mathrm{Ro}=\mathrm{K} / \mathrm{N})$, grain filling time (days), time of maximum grain filling ( $\mathrm{T}_{\max . \mathrm{G} \text {; day), weight at }}$ maximum grain filling time $\left(\mathrm{W}_{\max . \mathrm{G}} ; \mathrm{mg}\right)$ and growth $(\%)$ of the final growth at the time of maximum grain filling time (I\%) differed significantly among the treatments (Table 2). Average grain filling rate $\left(\mathrm{G}_{\text {avg }}\right)$ was found the highest (1.36 $\mathrm{mg} /$ day $)$ under the control. But maximum grain filling rate $\left(\mathrm{G}_{\max }\right)$ was found the highest $(1.69 \mathrm{mg} /$ day $)$ under high temperature. Time required for grain filling was longest (33.63 days) in low temperature and was shortest (23.74 days) in high temperature. The highest Initial growth potential $\left(R_{0}=K / N\right)$ value was found 
under high temperature (0.7355) and the lowest value was found under low temperature (0.3728). Time of maximum grain filling was longest (15.38 days) under low temperature and shortest (9.55 days) under high temperature. The highest weight at maximum grain filling time $\left(\mathrm{W}_{\max . \mathrm{G}} ; \mathrm{mg}\right)$ was found under high temperature $(13.85 \mathrm{mg})$ followed by control $(12.53 \mathrm{mg})$ and low temperature $(11.16 \mathrm{mg})$. Growth $\%$ of the final growth at the time of maximum grain filling time (I\%) was found highest under high temperature $(55.45 \%)$ and the lowest value (43.66) was found under low temperature. And an intermediate value of I\% was found in the control (47.61). Therefore, starch accumulation was faster under high temperature and slower under low temperature.

Table 2: Different starch accumulation parameter as influenced by 3 temperature regime.

\begin{tabular}{|c|c|c|c|c|c|c|c|}
\hline Treatment & $\begin{array}{c}\text { Average } \\
\text { grain } \\
\text { filling } \\
\text { rate } \\
\left(\mathrm{G}_{\text {avg }}\right) \\
(\mathrm{mg} / \mathrm{day})\end{array}$ & \begin{tabular}{|} 
Maximum \\
grain \\
filling \\
rate \\
$\left(\mathrm{G}_{\max }\right)$ \\
$(\mathrm{mg} /$ day $)$
\end{tabular} & $\begin{array}{c}\text { Initial growth } \\
\text { potential } \\
\left(\mathrm{R}_{0}=\mathrm{K} / \mathrm{N}\right)\end{array}$ & $\begin{array}{c}\text { Grain } \\
\text { filling } \\
\text { time } \\
\text { (days) }\end{array}$ & $\begin{array}{c}\text { Time of } \\
\text { maximum } \\
\text { grain } \\
\text { filling } \\
\left(T_{\text {max.G }}\right) \\
\text { (days) }\end{array}$ & $\begin{array}{c}\text { Weight at } \\
\text { maximum } \\
\text { grain filling } \\
\text { time } \\
\left(\mathrm{W}_{\text {max.G }}\right) \\
(\mathrm{mg})\end{array}$ & $\begin{array}{l}\text { Growth } \% \text { of } \\
\text { the final } \\
\text { growth at the } \\
\text { time of } \\
\text { maximum } \\
\text { grain filling } \\
\text { time, (I\%) }\end{array}$ \\
\hline $\begin{array}{l}\text { Low } \\
\text { temperature } \\
\text { treatment }\end{array}$ & 1.03 & 1.34 & 0.3728 & 33.63 & 15.38 & 11.16 & 43.66 \\
\hline $\begin{array}{l}\text { High } \\
\text { emperature } \\
\text { treatment }\end{array}$ & 1.24 & 1.69 & 0.7355 & 23.74 & 9.55 & 13.85 & 55.45 \\
\hline Control & 1.36 & 1.57 & 0.5924 & 27.72 & 12.52 & 12.53 & 47.61 \\
\hline $\operatorname{LSD}_{0.05}$ & 0.11 & 0.12 & 0.03 & 2.24 & 1.66 & 1.27 & 2.59 \\
\hline $\mathrm{CV}(\%)$ & 4.25 & 3.89 & 2.33 & 3.95 & 6.68 & 5.10 & 2.65 \\
\hline
\end{tabular}

\section{B. Discussion}

It is generally accepted that grain filling rate in cereals is mainly determined by sink strength. The sink strength can be described as the product of sink size and sink activity. Sink activity is a physiological restraint that includes multiple factors and key enzymes involved in carbohydrate utilization and storage (Liang et. al., 2001; Wang et.al., 2003). Starch consists of amylose (linear $\alpha-1,4-$ polyglucan) and amylopectin ( $\alpha$-1,6-branched polyglucans) in rice grains. Although amylose synthesis is exclusively governed by GBSS, amylopectin is synthesized via concerted reactions catalyzed by multiple isoforms of enzymes: SSS, SBE and SDBE (Nakamura, 2002; Tetlow, 2006). Temperature variation influences on the activity of enzymes as well as starch formation and finally affect on rice quality such as chalkiness. 
In this study, temperature regimes exhibited that SuSy activity was higher under high temperature treatments only at 7 DAF, but SuSy activity was lower after 7 DAF than the other two temperature treatments. From 14 DAF SuSy activity was higher under low temperature treatment than the other two temperature treatments. The relationship between chalkiness (\%) and SuSy activity was stronger and negative at significant level at 21,28 and 35 DAFs which indicates that higher SuSy activity under low temperature treatment at later grain filling stage plays an important role than the early to middle grain filling stage for reduction of chalkiness (\%). And the lower activity of this enzyme from the mid to the late grain filling stage under high temperature is the reason for higher chalkiness $(\%)$.

The present study showed that ADPG-Ppase activity was higher under high temperature treatment only at $7 \mathrm{DAF}$ than the other two temperature treatments but its activity was lower from 14 DAF in all the treatments. The loss of ADPGPpase activity under high temperature was rapid than the other two treatments. There was a significant positive correlation between the chalkiness (\%) and ADPG-Ppase activity at 7 DAF, but no correlation at 14 DAF and significantly negative correlation at 21, 28 and 35 DAFs, i.e., higher activity of ADPG-Ppase at the early caryopsis development stage and lower activity from the middle to the late caryopsis development stage under high temperature treatment caused higher chalkiness $(\%)$. On the other hand, higher activity of ADPG-Ppase under low temperature treatment from 14 DAF may be the reason for lower chalkiness (\%) due to sufficient ADP-glucose for starch synthesis. In maize, the activity of ADPG-Ppase was reduced by high temperature (Wilhelm et. al., 1999).

The initial activity of SSS under high temperature treatment was greater than the control and low temperature treatment, but after that the activity of SSS reduced rapidly and was lesser than the control and low temperature treatment which indicated that SSS is very much sensitive to temperature variation. The present result supports the previous report that the apparent rate for the SSS reaction in wheat, reached a maximum value between $20^{\circ} \mathrm{C}$ to $25^{\circ} \mathrm{C}$, above which the apparent rate fell as temperature was further increased (Jenner, 1994). The relationship between SSS activity and chalkiness (\%) was significantly positive only at the early stage of grain filling but significantly negative at 21, 28 and 35 DAF. Therefore, higher activity of SSS at the mid-late to the late caryopsis development stage mediated by low temperature reduced chalkiness(\%) and lower activity of SSS at the mid-late to the late caryopsis development stage mediated by high temperature increased chalkiness (\%).

The amylose content depends on GBSS activity because amylose is synthesized by only GBSS. GBSS activity was markedly higher under low temperature treatment throughout the grain filling stage followed by the control and high temperature treatment which implying that lower the temperature, increased the GBSS activity. This result attributed to the high temperature treatment resulted in 
a reduction of the activity and gene expression for GBSS in rice (Jiang et. al., 2003) and decrease of amylose content (Umemoto and Terashima, 2002). The correlation between GBSS activity and chalkiness (\%) was significantly negative during the whole grain filling period, but in the early and the late stage of grain filling, the activity of GBSS was not strongly correlated with chalkiness (\%). The stronger negative correlation between GBSS activity and chalkiness (\%) were observed at 14, 21 and 28 DAF which were $-0.8899^{* *},-0.9141^{* *}$ and $-0.8737^{* *}$ $(\mathrm{P}<0.01)$, respectively and this indicate that GBSS play a cardinal role to reduce chalkiness $(\%)$ in the mid to the mid-late stage of rice grain development.

The present experiment illustrated that SBE activity increased up to 14 DAF in all the three treatments and declined afterwards. Exposure to low temperature SBE activity was lower at the early grain development stage (7 DAF), but it's activity was the highest among the treatments from 14 DAF to 35 DAF. Under high temperature SBE activity was the highest only at 7 DAF. A significant and positive correlation was observed between chalkiness (\%) and SBE activity at 7 DAF but no significant correlation was found at 14 DAF and significantly negative correlation was found at 21,28 and 35 DAF which indicates that higher SBE activity under low temperature treatment at the later grain filling stage had a positive role in the reduction of chalkiness (\%) and lower SBE activity at the later stage of grain filling under high temperature treatment increased chalkiness (\%). Earlier report also showed that high temperature resulted in a reduction of activity and gene expression for SBEs in rice (Umemoto and Terashima, 2002).

The role of SDBE is remodeling of a soluble glucan precursor by the removal of certain branch linkages, thus facilitating the transition to the semicrystalline state (Streb et. al., 2008). From the early to the mid gain filling stage (up to 14 DAF) SDBE activity was higher under high temperature treatment over the control and low temperature treatment but at the later grain development stage SDBE activity decreased in the high temperature treatment over low temperature treatment and the control. The contrary trend was observed for low temperature treatment. In the present study a positive significant correlation was found between the chalkiness (\%) and SDBE activity at only 7 DAF but significant negative correlation was observed at the later stage of grain filling which implied that higher activity of SDBE under high temperature treatment at the early grain development stage and lower activity at the later grain filling stage were also responsible for increasing chalkiness (\%).

Average and maximum grain filling rate were the lowest under low temperature having longer grain filling time. Whereas, grain filling rate was maximum under high temperature having the shortest grain filling time. But, in control the average grain filling rate was highest having intermediate grain filling time. Besides, initial growth potential was the highest under high temperature. Therefore, starch accumulation rate was more rapid under high temperature, slowest under low temperature, and moderate under the control. Thus, 
temperature variation influenced on starch synthesis enzyme activities as well as head rice rate and chalkiness $(\%)$.

\section{Conclusion}

Significant differences were observed among the three temperature treatments on changes of chalkiness (\%), starch synthesis enzymes activity and also starch accumulation rate. High temperature increased grain chalkiness, reduced head rice rate and accelerated grain filling period and poor grain formation. The increased activity of Susy, ADPG-Ppase, SSS and SBE from the mid to the late grain filling stage and the higher activity of GBSS throughout the grain filling stage under low temperature treatment were closely associated with the reduction of rice grain chalkiness. And the contrary trends were observed for high temperature treatment. Therefore, rice grain chalkiness could be reduced by the regulation of starch synthesis enzymes activity in rice grains by genetic improvement.

\section{Acknowledgements}

This research work was financially supported by Program for the Improvement of Early Indica Rice Quality from The Ministry of Science and Technology (00NKY1002), National Natural Science Foundation of China (31570372), and National Agricultural Technology Program (NATP) phase-1, BARC, Dhaka, Bangladesh.

\section{References}

Borrell, Am., A. Garside and S. Fukai. 1997. Improving efficiency of water use for irrigated rice in a semi-arid tropical environment. Field Crops Res. 52(3): 231-248.

Chen, D. S., P. Cheng, D. H. Li and L. T Xiao. 2011. Studies on measurement system for rice chalkiness based on computer image processing. Journal of Hunan Agricultural University (Natural Sciences). 37(5): 469-473.

Dong, H., D. Z. Sang, P. Wang and J. C. Yang. 2006. Difference in chalky character of the grains at different positions within a rice panicle. Acta Agron.Sin. 32(1): 103111.

Douglas, C. D., M. K. Tsung and C. F. Frederick. 1988. Enzymes of sucrose and hexose metabolism in developing kernels of two inbreds of maize. Plant Physiol. 86: 10131019.

Gummert, M. 2010. Measuring white rice quality. Postharvest fact sheetof rice knowledge bank. International Rice Research Institute (IRRI), Los Banos, Philippines.

Han, X. Z. and B. R. Hamaker. 2001. Amylopectin fine structure and rice starch paste breakdown. J. Crop Sci. 34(3): 279-284.

Jenner, C. F. 1994. Starch Synthesis in the Kernel of Wheat under High Temperature Conditions. Aust. J. Plant Physiol. 21(6): 791-806. 
Jiang, H., W. Dian and P. Wu. 2003. Effect of high temperature on fine structure of amylopectin in rice endosperm by reducing the activity of the starch branching enzyme. Phytochemistry. 63(1): 53-59.

Keeling, P. L., J. R. Woods, R. H. Tyson and I. G. Bridges. 1988. Starch biosynthesis in developing wheat grain: evidence against the direct involvement of triose phosphates in the metabolic pathway. Plant Physiology. 87: 311-319.

Khush, G. S., C. M. Paul and La. N. M Cruz De. 1979. Rice grain quality evaluation and improvement at IRRI. In: International Rice Research Institute (ed). Proceedings of the workshop on chemical aspects of rice grain quality. IRRI, Los Banos, Philippines, pp. 390-392.

Koutroubas, S. D., F. Mazzini, B. Pons and D. A. Ntanos. 2004. Grain quality variation and relationships with morpho-physiological traits in rice (Oryza sativa L.) genetic resources in Europe. Field Crops Res. 86(2-3): 115-130.

Kubo, A., N. Fujita and K. Harada. 1999. The starch-debranching enzymes isoamylase and pullulanase are both involved in amylopectin biosynthesis in rice endosperm. Plant Physiol. 121: 399-409.

Liang, J., J. Zhang and X. Cao. 2001. Grain sink strength may be related to the poor grain filling of indica-japonica rice (Oryza sativa) hybrids. Physiologia Plantarum. 112(4): 470-477. doi: 10.1034/j.1399-3054.2001.1120403.x

Nakamura, Y. 2002. Towards a better understanding of the metabolic system for amylopectin biosynthesis in plants: rice endosperm as a model tissue. Plant and Cell Physiol. 43(7): 718-725.

Nakamura, Y., K. Yuki, S. Park and T. Ohya. 1989. Carbohydrate metabolism in the developing endosperm of rice grains. Plant and Cell Physiol. 30(6): 833-839.

Nelson, N. A. 1944. Photometric adaptation of the Somogyi method for the determination of glucose. J. Biol. Chem. 153: 375-380.

Richards, F. J. 1959. A flexible growth function for empirical use. Journal of Experimental Botany. 10(2): 290-301.

Somogyi, M. 1952. Notes on sugar determination. J. Biol. Chem. 195: 19-23.

Streb, S., T. Delatte. M. Umhang, S. Eicke, M. Schorderet, D. Reinhardt and S. C. Zeeman. 2008. Starch granule biosynthesis in Arabidopsis is abolished by removal of all debranching enzymes but restored by the subsequent removal of an endoamylase. Plant Cell. 20: 3448-3466.

Tetlow, I. J. 2006. Understanding storage starch biosynthesis in plants: a means to quality improvement. Can. J. Bot. 84: 1167-1185.

Tsai, C. Y. 1974. The function of the waxy locus in starch synthesis in maize endosperm. Biochemical Genetics. 11(2): 83-96.

Umemoto, T., Y. Nakamura and N. Ishikura. 1995. Activity of starch synthase and the amylose content in rice endosperm. Phytochemistry. 40(6): 1613-1616.

Umemoto, T. and K. Terashima. 2002. Activity of granule-bound starch synthase is an important determinant of amylose content in rice endosperm. Funct. Plant Biol. 29(9): 1121-1124. 
Venkateswarlu, B. and R. M. Visperas. 1987. Source-sink relationships in crop plants. Int. Rice Res. Paper Ser. 125: 1-19.

Wang, Xin, Ge. ZhiShen, Lei Cheng and Qing Yanli. 2003. Nurturing transgenic rice with soft rice varieties, Shanghai Academy of Agricultural Sciences. 19(2): 12-16.

Wardlaw, I. F. and J. Willenbrink. 1994. Carbohydrate storage and mobilisation by the culm of wheat between heading and grain maturity: the relation to sucrose synthase and sucrose-phosphate synthase. Aust. J. Plant Physiol. 21(3): 255-271.

Wilhelm, E. P., R. E. Mullen, P. L. Keeling and G. W. Singletary. 1999. Heat stress during grain filling in maize: effects on kernel growth and metabolism. Crop Sci. 39(6): 1733-1741.

Yoshida, S., D. A. Forno, J. H. Cock and K. A. Gomez. 1976. Laboratory manual for physiological studies of rice. 3rd ed. Philippines: International Rice Research Institute.

Zhang, H., S. Zhang, J. Yang, J. Zhang and Z. Wang. 2008. Postanthesis moderate wetting drying improves both quality and quantity of rice yield. Agron. J. 100(3): 726-734.

Zhu, Qingsen, Cao Xianzu and Luo Yiqi. 1988. Growth analysis on the process of grain filling in rice. Acta Agronomica Sinica. 14(3): 182-193. 